\title{
THE LELONG NUMBER OF A COMPLETE INTERSECTION
}

PAUL R. THIE

Let $A$ be an analytic set of pure dimension $p$ in a neighborhood of the origin in $C^{n}$, and assume that 0 is a point of $A$. The Lelong Number of $A$ at 0 , denoted by $n(0, A)$, is defined as the limit as $r$ goes to zero of the volume of $A$ in a ball of radius $r$, center 0 , divided by the volume of a $p$-dimensional linear subspace in such a ball (see [5]). Suppose now that $A$ is locally a complete intersection at 0 , that is, that there exists a neighborhood $U$ of 0 and $q=n-p$ functions $f_{1}$, $\cdots, f_{q}$ holomorphic on $U$ such that

$$
U \cap A=\left\{z \in U \mid f_{i}(z)=0, i=1, \cdots, q\right\} .
$$

Define $f: U \rightarrow C^{q}$ by $f=\left(f_{1}, \cdots, f_{q}\right)$. Then for any $z \in U$, codima $f^{-1}(f(z)) \leqq q$, that is, $\operatorname{dim}_{3} f^{-1}(f(z)) \geqq p$. On the other hand, by the upper semicontinuity of $\operatorname{dim}_{\mathfrak{z}} f^{-1}(f(z)),[2$, p. 46, Corollary 5], there exists a neighborhood $U^{\prime} \subset U$ of 0 such that $\operatorname{dim}_{z} f^{-1}(f(z)) \leqq \operatorname{dim}_{0}$ $f^{-1}(f(0))=p$ for all $z \in U^{\prime}$. Thus the map $f$ is of pure fiber dimension $p$ on $U^{\prime}$, and so the multiplicity of $f$ at 0 , denoted by $\nu(0, f)$, is well defined (see [4]).

It will be shown here that: first, $n(0, A) \leqq \nu(0, f)$; and second, if the germs at 0 of $f_{1}, \cdots, f_{q}$ generate the ideal of function germs at 0 zero on $A$, denoted by $I(0, A)$, then $n(0, A)=\nu(0, f)$. The first inequality will follow from some recent results of $\mathrm{R}$. Draper in [1], and the second from the use of a generalization of Rouchés Theorem, as proven by W. Stoll in [4]. The simple example in $C^{2}$ of $A=C \times\{0\}$ and $f(z, w)=w^{2}$ demonstrates that equality of $\nu(0, f)$ and $n(0, A)$ is not always possible. In the case of codimension one, the above results were first proven by Stoll in [3].

Theorem. Let $A$ be a pure p-dimensional analytic set in a neighborhood of the origin in $\boldsymbol{C}^{n}$, and suppose that 0 is a point of $A$. Let $f_{1}$ $\cdots, f_{q}$ be $q=n-p$ functions holomorphic in a neighborhood $U$ of 0 such that

$$
U \cap A=\left\{z \in U \mid f_{i}(z)=0, i=1, \cdots, q\right\} .
$$

Define $f=\left(f_{1}, \cdots, f_{q}\right): U \rightarrow C^{q}$. Then $n(0, A) \leqq \nu(0, f)$. Moreover, if the germs at 0 of $f_{1}, \cdots, f_{q}$ generate $I(0, A)$, then $n(0, A)=\nu(0, f)$.

Received by the editors June 9, 1969. 
Proof. Choose a coordinate system $\left(z_{1}, \cdots, z_{n}\right)$ of $C^{n}$ such that if $N=\left\{\left(0, \cdots, 0, z_{p+1}, \cdots, z_{n}\right) \in C^{n} \mid z_{i} \in C, \quad i=p+1, \cdots, n\right\}$, then $N \cap A$ and $N \cap T$ both contain 0 as an isolated point, where $T$ is the tangent cone to $A$ at 0 . Now from Theorem 7.3 of $[1], n(0, A)$ equals the degree of $A$ at 0 (as defined in [1]). Moreover by the choice of the coordinate system, from Theorem 6.3 of [1], it follows that the degree of $A$ at 0 equals $i(A \cdot N, 0)$, where $i(A \cdot N, 0)$ is the intersection multiplicity of $A$ with $N$ at 0 . Let $\pi: C^{p} \times C^{q} \rightarrow C^{p}$ be the projection map. Then in this case, $i(A \cdot N, 0)$ is simply the covering number $k$ of $\pi \mid A: A \rightarrow C^{p}$, that is, the degree of the Weierstrass polynomial associated with any regular coordinate system which has $z_{1}, \cdots, z_{p}$ as its first $p$ coordinates. Thus $n(0, A)$ equals the covering number $k$.

Now consider $\nu(0, f)$. By choice of $N$, from Theorem 7.1 of [1], it follows that $\nu(0, f)=\nu(0, f \mid N)$. Now Proposition 7.2 of [1] shall be applied. Let $0^{\prime}$ denote the origin in $C^{p}$ and $0^{\prime \prime}$ the origin in $C^{q}$. Choose open sets $U^{\prime}$ and $U^{\prime \prime}$ such that $0^{\prime} \in U^{\prime} \subset C^{p}, 0^{\prime \prime} \in U^{\prime \prime} \subset C^{q}$, and $U^{\prime} \times U^{\prime \prime} \subset U$. Define $\tilde{f}: U^{\prime} \times U^{\prime \prime} \rightarrow U^{\prime} \times C^{q}$ by

$$
\tilde{f}\left(\left(z_{1}, \cdots, z_{p} ; z_{p+1}, \cdots, z_{n}\right)\right)=\left(z_{1}, \cdots, z_{p}, f\left(z_{1}, \cdots, z_{n}\right)\right) .
$$

Then $\tilde{f}^{-1}\left(\tilde{f}\left(\left(0^{\prime}, 0^{\prime \prime}\right)\right)\right)$ contains $\left(0^{\prime}, 0^{\prime \prime}\right)=0$ as an isolated point, and so $\tilde{f}$ is light in a neighborhood of 0 by upper semicontinuity. Applying Proposition 7.2, comparing

\begin{tabular}{|c|c|c|c|c|c|c|c|c|}
\hline There & $M$ & $R$ & $N$ & $f$ & $q$ & $\tilde{f}$ & $a$ & $b$ \\
\hline Here & $U^{\prime \prime}$ & $U^{\prime}$ & $C^{q}$ & $f$ & 0 & $\tilde{f}$ & $0^{\prime \prime}$ & $0^{\prime}$ \\
\hline
\end{tabular}

it follows that $\nu\left(0^{\prime \prime}, f_{0^{\prime}}\right)=\nu\left(\left(0^{\prime}, 0^{\prime \prime}\right), \bar{f}\right)=\nu(0, \tilde{f})$, where $f_{0^{\prime}}: U^{\prime \prime} \rightarrow \boldsymbol{C}^{q}$ is defined by $f_{0^{\prime}}\left(z_{p+1}, \cdots, z_{n}\right)=f\left(0^{\prime}, z_{p+1}, \cdots, z_{n}\right)$. But by definition, $\nu(0, f \mid N)=\nu\left(0^{\prime \prime}, f_{0^{\prime}}\right)$. Hence $\nu(0, f)=\nu(0, f \mid N)=\nu(0, \tilde{f})$.

Choose a neighborhood $W \subset U$ of $0 \in C^{n}$ such that $\tilde{f}$ is light on $W$. Choose polydiscs $P$ and $Q, 0^{\prime} \in P \subset C^{p}, 0^{\prime \prime} \in Q \subset C^{q}$, such that

(i) $\bar{P} \times \bar{Q} \subset W$

(ii) $\left(\left\{0^{\prime}\right\} \times \bar{Q}\right) \cap A=\{0\}$

(iii) $(\bar{P} \times(\bar{Q}-Q)) \cap A=\varnothing$

(iv) There exists a thin analytic set $D \subset P$ such that for any $a \in P-D$, there exists exactly $k$ distinct points in $A \cap(P \times Q)$ with projection $a$. Moreover, these $k$ points are simple points of $A$ with the first $p$ coordinates providing local coordinates. Then by definition of $\nu(0, \tilde{f})$ and $\tilde{f}, \nu(0, \tilde{f})=\lim \sup ^{a \rightarrow 0} \#\left\{\tilde{f}^{-1}(\tilde{f}(\mathfrak{a})) \cap(P \times Q)\right\}$ 


$$
\begin{aligned}
=\underset{\mathfrak{a} \rightarrow 0}{\limsup } \#\left\{\left(a_{1}, \cdots, a_{p}, z_{p+1}, \cdots, z_{n}\right) \in P \times Q \mid \pi(\mathfrak{a})\right. \\
\left.=\left(a_{i}, \cdots, a_{p}\right) \text { and } f\left(a_{1}, \cdots, a_{p}, z_{p+1}, \cdots, z_{n}\right)=f(\mathfrak{a})\right\} .
\end{aligned}
$$

And

$$
\begin{aligned}
n(0, A)= & k=\limsup _{\mathfrak{a} \in A, \mathfrak{a} \rightarrow 0} \#\left\{\left(a_{1}, \cdots, a_{p}, z_{p+1}, \cdots, z_{n}\right)\right. \\
& \left.\in P \times Q \mid f\left(a_{1}, \cdots, a_{p}, z_{p+1}, \cdots, z_{n}\right)=0=f(\mathfrak{a})\right\} .
\end{aligned}
$$

Thus $n(0, A) \leqq \nu(0, \bar{f})=\nu(0, f)$.

To prove the second assertion, assume the germs of $f_{1}, \cdots, f_{q}$ at 0 generate $I(0, A)$. Shrinking the above neighborhoods $W, P$ and $Q$ if necessary, assume that they satisfy conditions $\mathrm{i}-\mathrm{iv}$ above and, moreover, that $f$ is of pure fiber dimension $p$ on $W$ and that, for any $\mathfrak{a} \in A \cap W$, the germs of $f_{1}, \cdots, f_{q}$ at a generate $I(\mathfrak{a}, A)$.

From Proposition 1.3 of [4], there exists an analytic set $T$ in $P \times Q$, thin in $P \times Q$, such that for any $\mathfrak{a} \in P \times Q-T$,

$$
\begin{aligned}
\nu(0, \tilde{f})=\#\left\{\left(a_{1}, \cdots, a_{p}, z_{p+1}, \cdots, z_{n}\right)\right. \\
\left.\quad \in P \times Q \mid f\left(a_{1}, \cdots, a_{p}, z_{p+1}, \cdots, z_{n}\right)=f(\mathfrak{a})\right\}
\end{aligned}
$$

where $\pi(\mathfrak{a})=\left(a_{1}, \cdots, a_{p}\right)$.

And there exists an open set $S \subset P \times Q$ containing 0 such that $f^{-1}(f(z)) \cap(\bar{P} \times(\bar{Q}-Q))=\varnothing$ for all $z \in S$. For if not, then there exists a sequence $\left\{z_{n}\right\}, z_{n} \rightarrow 0$, such that $f^{-1}\left(f\left(z_{n}\right)\right) \cap(\bar{P} \times(\bar{Q}-Q)) \neq \varnothing$. Assume $\mathfrak{x}_{n} \in f^{-1}\left(f\left(z_{n}\right)\right) \cap(P \times(\bar{Q}-Q))$. Then $\left\{\mathfrak{r}_{n}\right\}$ has a convergent subsequence, which will also be denoted by $\left\{\mathfrak{r}_{n}\right\}$. Let $\mathfrak{x}_{n} \rightarrow \mathfrak{r}_{0} \in \bar{P} \times(\bar{Q}-Q)$. But $f\left(\mathfrak{r}_{n}\right)=f\left(z_{n}\right) \rightarrow f(0)=0$. Therefore $f\left(\mathfrak{r}_{0}\right)=$ limit of $f\left(\mathfrak{r}_{n}\right)=0$. Therefore $\mathfrak{r}_{0} \in A \cap(\bar{P} \times(\bar{Q}-Q))$, a contradiction.

Since $f$ is of pure fiber dimension $p$ on $W, f \mid W$ is an open map [2, p. 132, Proposition 4]. Thus there exists an open ball $B \subset C^{q}$ with center 0 such that $B \subset f(S)$. Let $R=f^{-1}(B) \cap S$, an open neighborhood of $0 \in C^{n}$.

Take any $\mathfrak{a} \in R, \mathfrak{a} \notin \pi^{-1}(D) \cup T$. Let $\pi(\mathfrak{a})=a$. Choose $Q^{\prime}$ open in $\boldsymbol{C}^{q}$ such that $\{a\} \times \bar{Q} \subset\{a\} \times Q^{\prime} \subset W$. Define $I=[0,1]$ and $h: Q^{\prime} \times I \rightarrow C^{q}$ by $h\left(z_{p+1}, \cdots, z_{n} ; t\right)=f\left(a, z_{p+1}, \cdots, z_{n}\right)$. Define $g: I \rightarrow \boldsymbol{C}^{q}$ by $g(t)=t f(\mathfrak{a})$. Since $\tilde{f}$ is light on $W$, the map $h_{t}: Q^{\prime} \rightarrow \boldsymbol{C}^{q} \mathrm{de}-$ fined by $h_{t}\left(z_{p+1}, \cdots, z_{n}\right)=h\left(z_{p+1}, \cdots, z_{n} ; t\right)$ is light. Moreover for any $t \in I, h_{t}^{-1}(g(t)) \cap(\bar{Q}-Q)=\varnothing$ since $g(t) \in B$ and so $\left\{f^{-1}(f(z)) \mid z \in S\right\}$ 
$\supset\{a\} \times\left\{h_{t}^{-1}(g(t)) \mid t \in I\right\}$. Thus Rouche's Theorem, Theorem 2.7 of [4], can be applied. Compare

\begin{tabular}{|c|c|c|c|c|c|c|c|}
\hline There & $I$ & $M$ & $N$ & $m$ & $f$ & $g$ & $G$ \\
\hline Here & $I$ & $Q^{\prime}$ & $C^{q}$ & $q$ & $h$ & $g$ & $Q$ \\
\hline
\end{tabular}

The theorem states that

$$
\sum \nu\left(z, g(t), h_{t}\right) \text { is constant for } t \in I,
$$

and in particular,

$$
\sum \nu\left(z, f(\mathfrak{a}), h_{1}\right)=\sum \nu\left(z, 0, h_{0}\right),
$$

(where $\nu(x, y, F) \equiv 0$ if $F(x) \neq y$ and $\nu(x, F)$ if $F(x)=y$, and where all summations are extended over all $z \in Q)$.

First,

$$
\begin{aligned}
\sum \nu\left(z, f(\mathfrak{a}), h_{1}\right) \geqq \#\left\{\left(z_{p+1}, \cdots, z_{n}\right) \in Q \mid h_{1}\left(z_{p+1}, \cdots, z_{n}\right)=f(\mathfrak{a})\right\} \\
=\#\left\{\left(a, z_{p+1}, \cdots, z_{n}\right) \in P \times Q \mid f\left(a, z_{p+1}, \cdots, z_{n}\right)=f(\mathfrak{a})\right\} \\
=\nu(0, \tilde{f})=\nu(0, f) .
\end{aligned}
$$

Second, take any $z \in Q$ such that $h_{0}(z)=0$, that is, such that $f(a, z)$ $=0$. Then $z=(a, z)$ is a simple point of $A$ since $a \notin D$, and moreover, the function germs at $z$ of $f_{1}, \cdots, f_{q}$ generate $I(z, A)$. Therefore the rank of the Jacobian matrix of $f=\left(f_{1}, \cdots, f_{q}\right)$ at $z$ must be $q$. Hence, from Lemma 5.2 of $[4], \nu(z, f)=1$. Now let

$$
L=\left\{\left(a, z_{p+1}, \cdots, z_{n}\right) \mid z_{i} \in C, i=p+1, \cdots, n\right\} .
$$

Then $L \cap A$ contains $z$ as an isolated point. Moreover, since the first $p$ coordinates provide local coordinates to $A$ at $z$, it follows that $L \cap T^{\prime}$ also contains $z$ as an isolated point, where $T^{\prime}$ is the tangent cone equal to the tangent plane of $A$ at $z$. Thus $\nu(z, f)=\nu(z, f \mid L)$. But $f \mid L=h_{0}$, and so $\nu\left(z, 0, h_{0}\right)=\nu(z, f \mid L)=\nu(z, f)=1$.

Thus $\nu\left(z, 0, h_{0}\right)=1$ for all $z \in Q$ such that $\nu\left(z, 0, h_{0}\right) \neq 0$. And there are exactly $k$ points in $Q \cap A$ with projection $a$, that is, $k$ points in $Q$ such that $\nu\left(z, 0, h_{0}\right) \neq 0$. Therefore $n(0, A)=k=\sum \nu\left(z, 0, h_{0}\right)$ $=\sum \nu\left(z, f(\mathfrak{a}), h_{1}\right) \geqq \nu(0, f)$. Hence $n(0, A)=\nu(0, f)$.

\section{REFERENCES}

1. R. Draper, Intersection theory in analytic geometry, Math. Ann. 180 (1969), 175-204. 
2. R. Narasimhan, Introduction to the theory of analytic spaces, Lecture Notes in Math., no. 25, Springer-Verlag, Berlin, 1966. MR 36 \#28.

3. W. Stoll, Mehrfache Integrale auf komplexen Mannigfaltigkeiten, Math. Z. 57 (1952), 116-154. MR 14, 550.

4. - The multiplicity of a holomorphic map, Invent. Math. 2 (1966), 15-58. MR $35 \# 1832$.

5. P. Thie, The Lelong number of a point of a complex analytic set, Math. Ann. 172 (1967), 269-312. MR 35 \#5661.

\section{Boston College}

